The original publication is available at www.springerlink.com.

Abraham, R., Tribolet, J., Winter, R., Transformation of Multi-level Systems - Theoretical Grounding and Consequences for Enterprise Architecture Management, in: Proper, H., Aveiro, D., Gaaloul, K. (Eds.), Advances in Enterprise Engineering VII, Springer Berlin Heidelberg, 2013, pp. 73-87., http://dx.doi.org/10.1007/978-3-642-38117-1_6

\title{
Transformation of multi-level systems - theoretical grounding and consequences for enterprise architecture management
}

\author{
Ralf Abraham ${ }^{1}$, José Tribolet ${ }^{2,3}$, and Robert Winter ${ }^{1}$ \\ ${ }^{1}$ University of St. Gallen, Institute of Information Management, Mueller-Friedberg-Strasse \\ 8, 9000 St. Gallen, Switzerland, \\ ${ }^{2}$ CODE, Center for Organizational Design \& Engineering, INOV, Rua Alves Redol 9, \\ Lisbon, Portugal, \\ ${ }^{3}$ Department of Information Systems and Computer Science, Instituto Superior Técnico, \\ Technical University of Lisbon, Portugal \\ \{Ralf.Abraham, Robert.Winter\}@unisg.ch, \\ Jose.Triboleteinesc.pt
}

\begin{abstract}
In this paper, we investigate the support of enterprise architecture management (EAM) for enterprise transformation. Conceptualizing enterprises as systems, we draw on two theories that investigate static and dynamic system aspects, respectively - the theory of hierarchical, multi-level systems and control theory. From the theory of hierarchical, multi-level systems, we first introduce three orthogonal dimensions of hierarchy - layers, strata, and echelons. We then position EAM as a cross-dimensional transformation support function in this there-dimensional hierarchy space. Finally, we draw on control theory to derive a model of control and feedback loops that enables a designed EAM support of system-wide transformations. Using this model, we propose to extend the multi-level systems theory by a set of interlinked feedback loops as a fourth dimension. A case study of transformation in the Portuguese air force serves as an example illustrating the usefulness of the two theories for describing enterprise transformation.
\end{abstract}

Keywords: Enterprise Architecture Management, Control, Feedback, MultiLevel Systems, Hierarchy

\section{Introduction}

Increasing variety in their environment forces enterprises to change themselves at an ever higher pace. Sources of variety in an enterprise's environment include economic pressures from competitors, as well as politically, socially or technologically-induced changes. We understand enterprise transformation as designed and fundamental 
change, in contrast to ad-hoc, routine change. Enterprise transformation is a purposeful steering intervention into an enterprise's evolution, in order to respond to perceived opportunities, deficiencies or threats [1]. Despite the relevance of enterprise transformation, a big number of these transformation efforts fail. Reports indicate failure rates ranging from 70 to 90 per cent, across a broad range of domains [2]. These failures are often traced back to mistakes in strategy implementation and the coordination of the actual transformation efforts. In order to successfully implement enterprise transformation, Dietz and Hoogervorst [2] argue that a constructional, white-box understanding of enterprises is required in addition to a functional, blackbox understanding.

One approach that is concerned with an understanding of enterprise construction is enterprise architecture (EA). The purposeful design and change of EA according to strategic goals is the concern of enterprise architecture management (EAM). By defining principles to restrict design freedom (and thereby guiding design), one of the core tasks of EAM is to coordinate enterprises transformation $[3,4,5]$. Since EA focuses both on results (e.g., models) and activities (e.g., principles) in designing an enterprise, we draw on two theories that focus on static and dynamic system aspects: The theory of hierarchical, multi-level systems and control theory. When applied to enterprises, the former theory is concerned with enterprise construction, and the latter with enterprise transformation.

Within his theory of hierarchical, multi-level systems, Mesarovic [6] distinguishes between three orthogonal notions of hierarchy: Strata, layers, and echelons. By following this explicit distinction, we are able to define the positioning of EAM in an enterprise by analysing it from multiple hierarchy angles. We aim to show that in order to support enterprise transformation, EAM must employ a more differentiated understanding of hierarchy. To break down the resulting three-dimensional hierarchy space (strata, layers, and echelons) to the specific purpose of describing enterprises, we will provide three exemplary organizational design and engineering (ODE) approaches that each focus on one dimension of hierarchy in particular. We then position EAM in this framework and show how it cuts across these hierarchical dimensions.

Having an understanding of the static aspects of enterprises and the positioning of EAM, we describe an enterprise from a dynamic perspective as comprising three different kinds of feedback loops. We identify enterprise transformation as a special instance of a feedback loop, as purposeful and designed change in contrast to both permanent, evolutionary adaptation and sudden improvisation. The research questions we address are the following:

1. How can a conceptualization of enterprises as hierarchical, multi-level systems improve the effect of EAM for their transformation?

2. How can transformation be grounded on multi-level systems theory and control theory?

The rest of this paper is organized as follows. Section 2 introduces the theory of hierarchical, multi-level systems, basics of EAM and provides a framework of hierarchy and a positioning of EAM. Section 3 discusses the concept of feedback loops. In sec- 
tion 4, a case study of the Portuguese air force illustrates both theories applied to a real organization. Section 5 discusses implications for EAM and offers a conceptualization of feedback loops as another dimension of hierarchy in Mesarovic's [6] terms. Section 6 summarizes related work, before section 7 discusses limitations and provides a conclusion.

\section{Framework of Hierarchy}

\subsection{Theory of hierarchical, multi-level systems}

To establish a framework for describing enterprises, we must first distinguish two contexts of hierarchy: In a management context, hierarchy means a relationship of authority and responsibility between higher and lower level units. This implies that higher level units have authority to delegate tasks to lower level units (which are required to carry out these tasks), but they must at the same time bear responsibility for their actions. Therefore, the concepts of authority and responsibility are inextricably linked. By contrast, in an engineering context, hierarchy refers to a vertical decomposition of a system into subsystems (i.e., a vertical arrangement of subsystems).

Hierarchy is primarily used in the latter context by Mesarovic [6] in his theory of hierarchical, multi-level systems. Mesarovic distinguishes three orthogonal notions of hierarchy to describe a system: Strata, layers, and echelons. Strata and layers refer to a vertical decomposition of the system (i.e., a decomposition of the overall system into subsystems), while echelons are a horizontal decomposition to coordinate and integrate activities of various decision units. To refer to any of these notions, the generic term 'level' is used. Fig. 1 is adapted from Mesarovic [6] and shows the three different notions of hierarchy combined to describe an enterprise. Note that both strata and layers can be used to decompose the entire system, but also to decompose the subsystem that is under the control of a certain decision unit. 


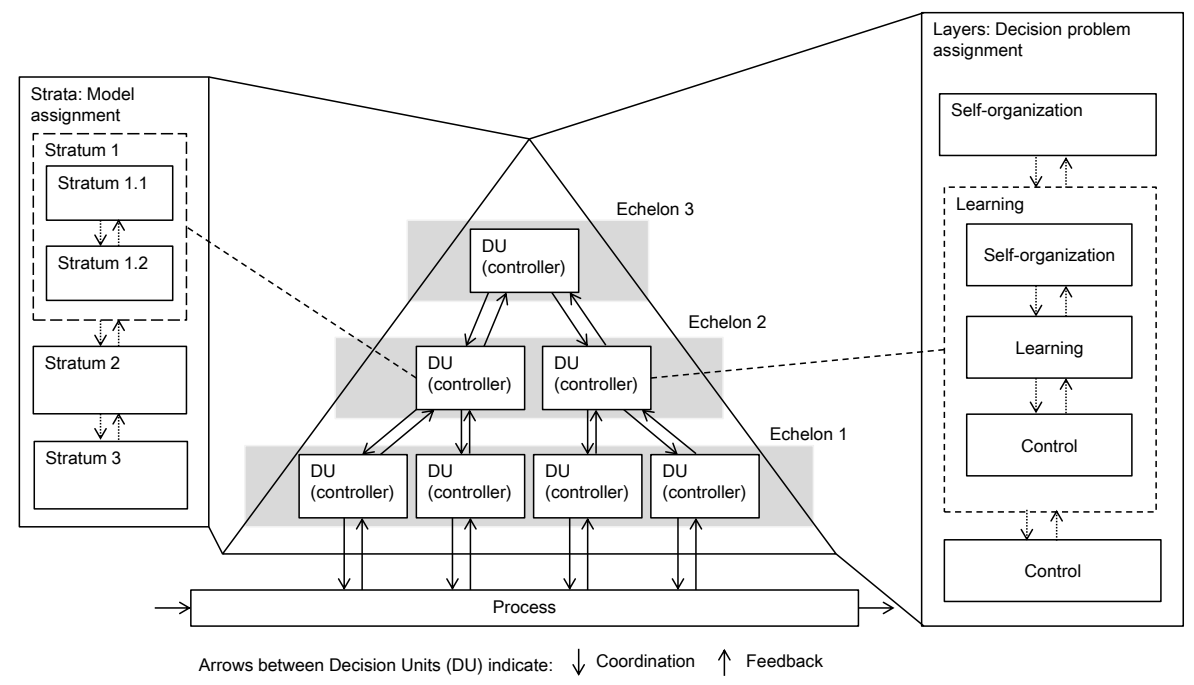

Fig. 1. Relationships of hierarchy notions in multi-level system (adapted from Mesarovic [6])

Despite the differences between the three notions of levels, there are some important commonalities: (1) Higher-level units are concerned with larger chunks of the overall system than lower-level units. (2) Higher-level units are concerned with the slower aspects of system behaviour; i.e. they are taking fewer decisions and intervene into system behaviour less frequently. (3) Problem descriptions on higher levels contain more uncertainty and are harder to formalize quantitatively than problem descriptions on lower levels.

The first notion of hierarchy, strata, describes levels of problem description or abstraction. While a model on a higher stratum provides a better understanding of overall system behaviour, a model on a lower stratum provides a more detailed functional explanation of individual system parts. What is considered as a whole system on a lower stratum may form a subsystem on a higher stratum. Strata thus describe the same entity on different levels of granularity. They may use a different form of modelling on each level, to capture the model contents in a suitable way.

The second notion of hierarchy, layers, describes levels of decision complexity. Since decision problems on lower layers can be expressed by more formal methods of description in the strata dimension, more formal techniques can be used to solve these problems. For example, in a manufacturing plant, a decision problem concerning optimum production sequences may be addressed by numerical optimization techniques (search layer), whereas decision problem on higher layers, such as which products to offer and how to market them (self-organizing layer), rely on less formal techniques such as heuristics. Layers reflect a hierarchy of goals, where the solution of goals on a higher layer decreases uncertainty on underlying layers. Put another way, lower layers need higher layers to limit their solution space - decisions made on higher layers limit the degree of design freedom on lower layers.

The third notion of hierarchy, echelons, is specific to organizations. Mesarovic [6] refers to echelons as a horizontal decomposition and calls the resulting system an 
organizational hierarchy. The term "organizational hierarchy" implies the use of hierarchy in the management context. On each echelon, there are one or more decision units. These decision units may be individuals or groups of individuals (e.g., an individual acting as project manager or an architecture board). As with the other notions of hierarchy, higher echelons are concerned with larger system aspects than lower echelons. The task of a given echelon may be represented by a stratified description of the subsystem under its control (from the echelon's perspective, this is the system, whereas from the overall system's perspective, this is a subsystem). Communication between echelons takes the form of coordination and performance; higher echelons coordinate lower echelons, whose performance can be interpreted as feedback to the higher echelons. Coordination from higher-level units precedes feedback from lowerlevel units. Consequently, success (achieving its goals) of higher echelons is dependent on the performance of lower echelons

\subsection{Enterprise Architecture Management}

According to the ISO/IEC/IEEE Standard 42010, architecture is defined as "the fundamental organization of a system, embodied in its components, their relationships to each other and the environment, and the principles governing its design and evolution" [7]. This definition of architecture involves two aspects: The first part of the definition forms a descriptive aspect, concerning the structure of the system's building blocks and the relationships between them. The second part ("[...] the principles [...]") forms a prescriptive aspect, effectively restricting the design and evolution space of the system under consideration. EAM is a continuous management process concerned with establishing, maintaining and purposefully developing an enterprise's architecture [8,9]. From the IEEE definition, architecture is concerned both with results (descriptive aspect) as well as with guiding activities leading to these results (prescriptive aspect). In terms of artifacts, EAM provides models on the current state of an enterprise (as-is), the future state (to-be), as well as a transition plan on how to get from the current to the future state.

Addressing the descriptive aspect of architecture, EAM is concerned with establishing transparency. Capturing the current state of EA and keeping this information up-to-date is therefore seen as one of the EAM team's core tasks $[8,10]$. Concerning the prescriptive aspect of architecture, EAM is concerned with maintaining consistency. Principles guide enterprise evolution by restricting design freedom [2] in order to maintain consistency between the enterprise strategy and its implementation (i.e., the actual EA). The management function of EAM is a good example to illustrate the interplay between strata, layers, and echelons:

By focusing on a high level of abstraction (high stratum) in the descriptive aspect, EAM is able to provide a holistic overview of the enterprise. In order to understand individual aspects of an enterprise in greater detail (e.g., the technical infrastructure), partial architectures have to be relied upon. This approach is referred to as "broad instead of deep": EAM cuts across several decision layers in an enterprise and provides suitable models of each layers' concerns on a high stratum. Examples of deci- 
sion layers may be found in the business engineering framework [11], ranging from strategy, organisation, alignment to software and infrastructure layers.

In the prescriptive aspect, EAM is concerned with restricting design freedom by providing architectural principles. EAM principles support a layer notion of hierarchy as described by Mesarovic: Decisions made on one layer restrict the search and solution space of lower layers. For example, a principle advocating the use of commercial off-the-shelf software on the organisation layer narrows down the solution space on the software layer by removing the option of in-house development (and the need to select a particular programming language of platform).

Finally, echelons describe governance hierarchies - which organizational entities have the right to make which decisions, and thus are able to influence the actions of organizational entities on a lower echelon. The cascade of architectural principles can therefore be seen as a representation of the organizational governance hierarchy: Who has the right to govern whose decisions - which principle owners (organizational units responsible for the formulation, justification and maintenance of a given principle) may restrict the design freedom of other organizational units.

\subsection{Framework of hierarchy and positioning of EAM}

To create a framework for EAM positioning, we first consider three ODE approaches that each illustrate one of the hierarchy notions previously discussed.

For the strata dimension, consider the architecture landscapes in the TOGAF framework [12, p. 481]. TOGAF contains architectural views on the enterprise with varying levels of granularity, namely strategic architecture describing a long-term, highly aggregated view on the enterprise, segment architecture focusing on a more detailed description of areas within the enterprise, and finally capability architecture to describe operational competencies.

We illustrate the layer dimension using the aspect organizations found in DEMO [13]. Enterprises are regarded on three different aspect organizations, namely the business organization (B-Organization), the intellect organization (I-Organization) and the document organization (D-Organization). These organizations form a hierarchy of decision layers, with higher layers setting a frame for lower layers and services from lower layers supporting the operation of higher layers: A redesign of the BOrganization results in changes to the I- and D-Organization. Bottom-up, the Dorganization supports the I-Organization, which in turn supports the B-Organization.

The echelon dimension is exemplified by the total information systems management (TISM) approach [14]. Management of information systems is broken down to five levels. Strategic guidelines, IS framework, IS Project Portfolio, IS Project and IS support. We will leave out IS support since this level focuses on local user support only instead of enterprise-wide aspects.

Each of these echelons is represented by organizational actors carrying out assigned roles with authority and responsibility. Organisational actors may either be individuals in roles or groups of individuals acting as boards. We shall call the area of authority and responsibility of organizational actors their domain. For example, the management board defines strategic guidelines, an architecture board defines the IS 
framework (in Österle et al.'s [14] definition, an IS framework covers not only data and functions of electronic information processing, but also the organizational dimension. This is in line with the notion of EAM extending beyond IT to also include business aspects), a project portfolio management board sequences individual projects, and finally project management teams carry out individual projects that generate new or improve existing capabilities.

We position EAM as shown in Fig. 2 on the highest stratum, the strategic architecture level. Lower strata are covered by detailed architectures such as segment or capability architectures. This is consistent with the idea that models on higher strata provide an explanation of the overall system behaviour, while lower-level models such as segment or capability architectures provide a more detailed functional explanation of subsystems.

While all organizational actors take part in EA (by creating organizational reality within their domain, where they enjoy freedom of action), the purposeful evolution of EA (i.e., its management: EAM) is the main task of a specific echelon, the EAM board. By using architectural principles to limit design freedom, EAM is a cross-layer approach. When principles are operationalized as concrete standards, they are used to guide enterprise design across all layers, ensuring that lower layers support higher layers.

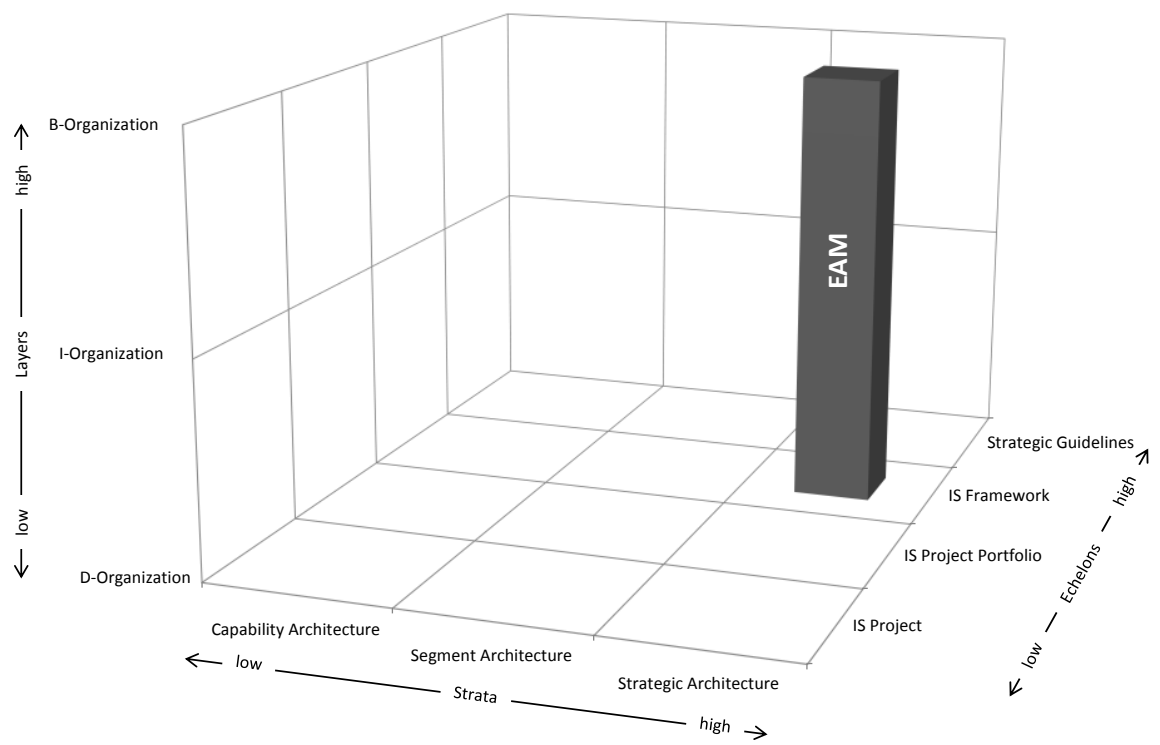

Fig. 2. Positioning of EAM in three-dimensional hierarchy space

\section{FEEDBACK LOOPS}

In order to describe dynamic aspects of a system, we turn to control theory and feedback loops [15]. Control is defined by Åström and Murray as "the use of algorithms 
and feedback in engineered systems" [15]. Feedback is a key component in improving a system's robustness against uncertainty. A simple example would be a feedback system to control the speed of a car, e.g. when cruise control is turned on. The actual speed of the car is observed by a sensor, and if deviations are sensed, the flow of petrol to the engine is regulated. Fig. 3 illustrates this example of a feedback loop with an observer (Sense Speed), a modeller (Compute) and a controller (Actuate Throttle).

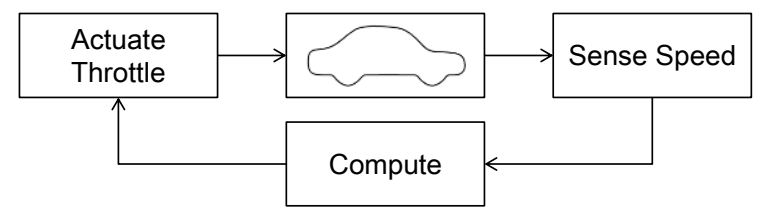

Fig. 3. Example of a feedback loop from Åström and Murray [15, p. 18]

By constantly observing system states and comparing observed data against system goals, a controller component can compute corrective measures and then change system variables to re-align to system goals. A feedback loop consists of an observer that records environmental data, a modeller that interprets the data and calculates corrective actions, and a controller that influences the system based on the input from the modeller. Thus, the actual car speed can be kept as close to the desired speed as possible. The example also shows that a system being observable is necessary for the system to be controllable: If the car speed cannot be sensed, it cannot be controlled. However, being observable is not sufficient for being controllable: If only the sensor worked, but not the components that compute speed adjustments and actuate the throttle, the car would still be uncontrollable. Furthermore, not all observable variables are also controllable.

In a complex system, there may be several feedback loops operating in parallel: Next to the feedback loop concerned with the car's speed, there are also several other loops, e.g. for climate control, regulating the air condition in the passenger cabin.

Like a travelling car, an enterprise can also be considered a system in which several feedback loops run in parallel. This theme of control is also central to one of the understandings of management: That as the structuring, control and development of productive social systems such as enterprises [14, p. 22]. Note that there are also other understandings of management, such as the behavioural notion that focuses on getting activities done by people. However, for the purpose of this paper, we will follow the notion of management as a cyclic feedback loop that comprises the activities plan, do, check, and act. Fig. 4 illustrates a hierarchical structure of three feedback loops. 


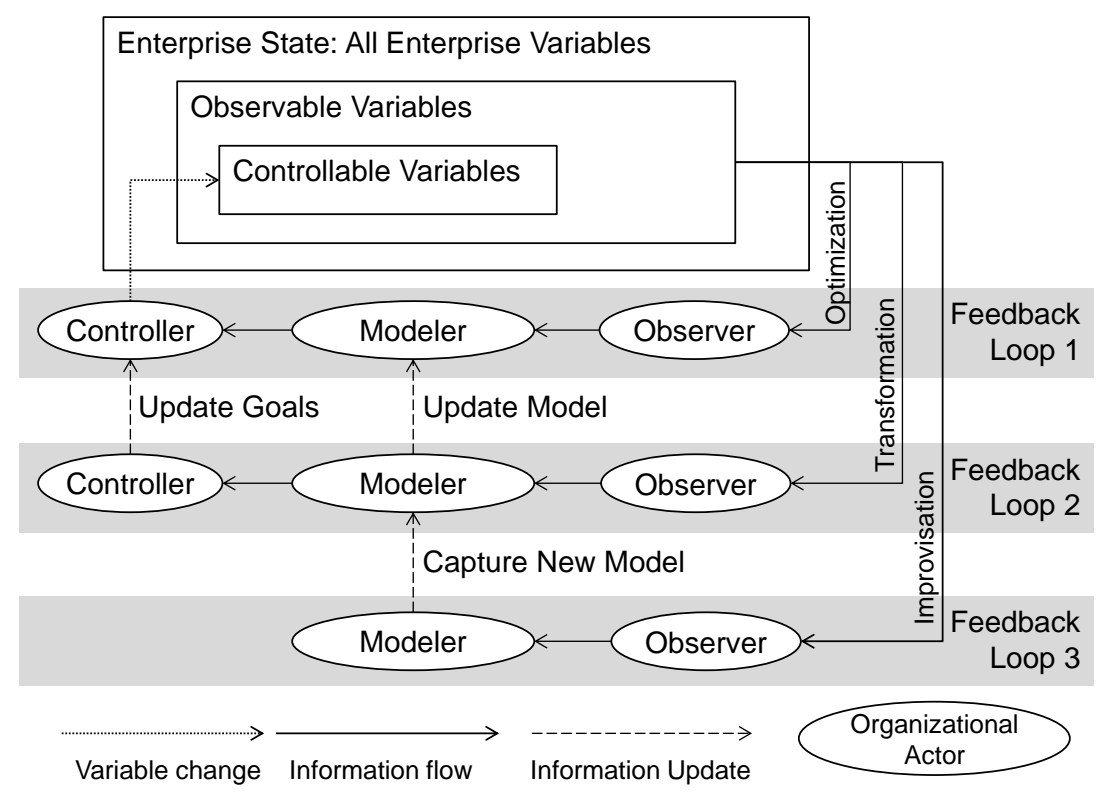

Fig. 4. Feedback loops in enterprises

Management in general, and also management of EA, is mandated by the fact that enterprises are never in stasis, but are constantly subjected to environmental turbulences. Three dimensions of environmental turbulence are suggested [16, 17]: Frequency, Amplitude, and Predictability. We consider an enterprise as having a certain state at each moment in time, and consisting of a set of observable and a set of controllable variables. Depending on the degree of environmental turbulence the enterprise encounters, one or several of the following feedback loops are triggered.

Feedback loop 1 is the basic loop that runs continuously. It deals with on-going optimization, with running the business. Turbulences occur frequently, but with low amplitude, and they are predictable. Based on existing models (e.g., architectural and process models), deviations are observed by organizational actors and control measures are initiated. These models describe the enterprise on different strata. It is important to note that all organizational actors run this first feedback loop within their own domains. Taking the example of a car company, inventory management or production sequencing on the assembly floor would be exemplary activities in feedback loop 1, as would be running IT systems in the administrative departments. In feedback loop 1, enterprises react to expected exceptions. This is also discussed as resilience by Aveiro et al. [18].

Feedback loop 2 is triggered when unexpected exceptions or environmental turbulences cause a change in models. In this case, turbulences occur less frequently than in feedback loop 1, but they have higher amplitude. Still, they are predictable and thus allow for initiating a planned, purposeful transformation. Therefore, enterprise transformation takes place in this loop. Enterprises continue running their business (i.e., conducting daily operations within feedback loop 1), but they take additional 
measures to transform themselves: They enter feedback loop 2, formulate transformation goals and initiate a series of projects that will eventually change the enterprise state (Update Model / Update Goals). When transformation is complete, enterprises exit feedback loop 2 and continue running feedback loop 1 which now operates on a new enterprise. That is, at the end of feedback loop 2, enterprises have reconfigured their operational resources to achieve a fit with the new environment [19]. They have also updated their models to reflect this new environment. The development of a new generation, fuel-saving engine would be a concrete example in a car company.

Feedback loop 3 is triggered when an enterprise is faced with environmental turbulences that require immediate reactions. These turbulences are characterized by high amplitude and virtually no predictability [20]. As opposed to feedback loop 2, environmental change in this feedback loop is unpredictable and therefore requires quick, improvisational actions that result in the capture of a new model [21]. Once an enterprise has made sense of the new situation (i.e., models and goals have been updated), it goes back to feedback loops 2 and 1 in order to initiate the necessary transformation projects and continue running its business.

After feedback loops 2 or 3 have handled unknown exceptions for the first time, these exceptions are no longer unknown and can in the future be dealt with by a lower feedback loop, e.g. by feedback loop 1. In other words, enterprises that have experienced certain environmental turbulences may learn from these experiences if they are able to add them to their pool of known exceptions $[18,19]$. If enterprise goals were never questioned, and enterprise models never updated, enterprises would be limited to single-loop learning. A hierarchy of feedback loops allows for updating enterprise goals and models and enables double-loop learning in organizations [22]: modifying variables based on previous experiences.

The following example may illustrate the transitions between different feedback loops and the potential for organizational learning: Consider a car company is hit by a strike from one of its suppliers. It may try to respond to this situation within feedback loop 1, by attempting to secure the required material from another source. If this fails, it may enter feedback loop 2, starting negotiations with unions to reach a settlement. If this also fails, it may enter feedback loop 3 and try to change its entire logistics from just-in-time supply to in-stock supply. In this case, the company may no longer be threatened by further exceptions of the same kind (strikes) - this kind of exception is from now on included in the pool of known exceptions and can in the future be dealt with by feedback loop 1 .

\section{Case Study: Transformation in the Portuguese Air Force}

To give a practical example of feedback loops and different notions of hierarchy describing an enterprise transformation, we consider the example of the Portuguese air force case study as reported by Páscoa and Tribolet [23]. This organization operates different weapons systems that can be characterized by their degree of technological advancement. In normal operating mode, the mission of the air force includes the defence of the national airspace, air transport operations, as well as search and rescue 
missions. Its core business process is flying. Being a non-profit organization dependent on state funding, the Portuguese air force is developing a strategy map to maximize its core output - the number of flying hours - given a fixed annual budget. The overall goal is broken down into individual objectives like improving drafting of personnel, or providing more efficient aircraft maintenance processes.

Considering the strata notion of hierarchy, the Portuguese air force has developed a number of models like an objectives model (providing a mapping of objectives to business models), descriptions of business processes (e.g. flight operation, personnel training, and public relations), or performance indicators and dashboards providing information on various organizational subsystems (e.g., describing the number of people involved with a specific weapon system or the number of missions performed by given air craft or squadrons). Using this stratified description, several scenarios (organizational configurations) can be described to identify the impact of possible budget cuts.

On the layer dimension, a set of business rules and policies has been established in order to guide the implementation of the overall goals. These policies and business rules can be interpreted as architectural principles, allowing commanding units (the organizational echelons) to restrict the design freedom of their subordinates.

From the dynamic point of view, on-going flight operations, search and rescue missions, and defence readiness are controlled by feedback loop 1, representing the air force's daily business. The strategic initiatives leading to the formulation of the objectives model relate to feedback loop 2. They represent a planned change of the business, a new organizational configuration that is being designed (the equivalent EA term would be a to-be model) and that eventually replaces the existing configuration (the as-is model). In order to guide the change in organizational configurations, the Portuguese air force is developing a strategy map that represents the transition path considered most feasible to reach the given goals and objectives. Feedback loop 3 would be entered if the organization was faced with extreme environmental turbulences, such as a coup d'état.

The case study particularly points out the importance of organizational selfawareness, i.e. the idea that in order to perform any kind of control, system variables need to be observed. To this end, a wide array of indicators and dashboards has been developed to provide live information on the organizational status. These indicators cover different levels of description, i.e. the information contained in them describe the overall system on different strata, from flight operations of individual squadrons to overall organizational issues like budget availability for certain aircraft or technology components. Concrete steps towards increasing organizational self-awareness include the introduction of a formally agreed-upon terminology throughout the air force, moving from paper-based to electronic document storage and retrieval in order to increase dissemination and availability of information, uniform definition of roles (qualification, authority and responsibility) across the organization, and the continuing introduction of metrics. Metrics can be used to demonstrate to individual actors their contribution to the overall goals and objectives of the air force, as well as to observe more system variables and thus reduce the latency of the feedback loops. 


\section{Discussion}

As discussed in section 3, the notion of feedback is central to both running the business (feedback loop 1) and changing the business (feedback loop 2). Only when system variables are observed can they be controlled. In the case of EAM, the architecture team may supply models to describe the system on different strata, but the contents of these models are provided by all organizational actors. This is because organizational actors must be provided with a degree of freedom to act within their domains, otherwise an enterprise would lose the ability to react to exceptions. EAM therefore depends on the existence and use of feedback channels that allow each organizational actor to act as observers, detecting model changes within their domain and propagating this data into models. Therefore, model updating mechanisms are central to EAM. If the observer or modeller part in any feedback loop fails, then the system cannot be purposefully controlled or transformed. A concrete example of an update mechanism is provided by Castela et al. [24].

In the case of EA principles, there is also interplay between coordination from higher echelons to lower echelons, and feedback in the other direction. Existing EA principles (i.e., principles based on existing models) can be used to run feedback loop 1. Known exceptions that might occur in this loop can be dealt with within existing principles. However, when feedback loops 2 or 3 are entered, information must be fed back in order to adapt principles to the new environmental situation. For example, a principle in a bank stating that all development activities are to be performed using a certain programming language works well as long as the environment remains stable and the enterprise remains in feedback loop 1. However, when transformation occurs, and a banking application has to be offered on mobile devices that cannot be supported by the existing programming language, these principles need to be adapted to the new model [25]. While all organizational actors are responsible for adhering to principles in their domain, they also need to challenge the validity of existing principles when the environment changes (i.e., when feedback loops 2 or 3 are triggered). Therefore, organizational actors on all echelons take part in governance.

The application of control theory adds semantics to the understanding of the enterprise that is not covered by the original three dimensions of the hierarchical systems theory. The commonalities between all notions of hierarchy identified in section 2 also hold for a hierarchy of feedback loops:

(1) Higher feedback loops are concerned with larger aspects of the overall system than lower feedback loops. Feedback loop 1 deals with running the business. Many instances of feedback loop 1 run in parallel, but each one is performed for smaller chunks of the system. Feedback loops 2 and 3 are triggered by exceptions that concern larger system aspects.

(2) Running frequency decreases from feedback loop 1 to feedback loop 3. While feedback loop 1 runs permanently, feedback loops 2 and 3 are triggered by unknown exceptions in the environment and therefore run less frequently. Feedback loop 3 is triggered by unpredictable, sudden exceptions and requires improvisation. This occurs less frequently than predictable exceptions leading to purposeful transformation in feedback loop 2 . The decrease in running frequency as one moves up in levels is also 
emphasized by the fact that previously unknown exceptions are added to the pool of known exceptions after they have successfully been handled.

(3) Problem descriptions on higher feedback loops are harder to formalize and contain a greater degree of uncertainty than problem descriptions on lower feedback loops. This is also consistent with the concept of rising environmental turbulence triggering unexpected exceptions and therefore being addressed by higher feedback loops.

We therefore conclude that feedback loops extend the three original dimensions of the theory of hierarchical systems as a fourth dimension. This fourth dimension forms a vertical, hierarchical decomposition of downward control and upward feedback flows in the system, analogously to strata focusing on problem description and layers focusing on goal decomposition. Higher levels in the strata dimension imply higher degrees of abstraction. Higher levels in the layer dimension imply less formalized decision goals. Higher levels in the feedback loop dimension imply a rising degree of environmental turbulence that leads to the occurrence of new, unknown exceptions in the enterprise. As a consequence, we formulate the following proposition:

P1: The three feedback loops specified in section 3 can be regarded as levels of an additional hierarchy dimension in the sense of Mesarovic's theory, with feedback loop 1 occupying a lower level and feedback loop 3 a higher level.

The dimension of feedback loops is orthogonal to the other three dimensions, in that it can be applied to the overall system or to certain subsystems, as can the other hierarchical notions of strata and layers [6]. Organizational actors as decision units on a given echelon run through this hierarchy of feedback loops to address problems in their domain. In a similar fashion, they use a number of layers to break down their decision problems into sub-goals and a number of strata or describe it on various levels of abstraction. Feedback loops add dynamic aspects, describing an enterprise at runtime that handles environmental influences. The original dimensions of hierarchy: strata, layers, and echelons concern static system aspects. Compare feedback loops to the governance hierarchy: While governance describes a static allocation of decision rights, authority and responsibility, feedback loops show how different governance entities interact and self-activate during different degrees of environmental turbulence. Regarding both static and dynamic system aspects is consistent with a view on EAM as not merely a passive, documentation-oriented exercise, but as an ODE approach that actively support and guides organizational design.

\section{$6 \quad$ Related work}

Fundamental to the application of both the theory of hierarchical, multi-level systems and control theory is the conceptualization of enterprises as dynamic systems. For modeling static aspects of enterprises, modeling approaches such as DEMO focus mostly on the stratum dimension of enterprises, and methods like UML lack a theoretical foundation. As we are specifically interested in providing a clear distinction be- 
tween different types of hierarchy, we chose the theory of hierarchical, multi-level systems to describe the composition of an enterprise.

For modeling dynamic aspects of enterprises, there are several approaches: For example, the Deming cycle [26], a four-step iterative management method, consisting of the phases of plan-do-check-act. This cycle can also be seen as a sequence of feedback loops (observe-model-control).

The Dynamic capabilities framework [21, 27] emphasizes a reconfiguration of an enterprise's operational structure in order to achieve a better fit with the environment. Dynamic capabilities such as business process management, research and development, or EAM all emphasize planned, structured transformation (contrasted to spontaneous, improvisational transformation), which is why maintaining a dynamic capability in an enterprise is associated with additional effort [28].

Beer's viable systems model (VSM) [29] describes autonomous systems that are able to survive in a changing environment. It can be used to describe how enterprises are affected by and react to environmental (i.e., market) dynamics. The VSM consists of five levels, where the first three levels (primary activities, communication, and control) are responsible for running the business, and the last two levels (environmental scanning and policy decisions) are responsible for changing the business.

We chose control theory because it provides the ability to model the connections between running and changing the business - optimization versus transformation via multiple levels of connected feedback loops, and because of its fit with the theory of hierarchical, multi-level systems.

\section{Conclusion}

In this paper, we have provided a theoretical grounding for enterprise transformation on multi-level systems theory and control theory. We have also conceptualized a multi-level structure of feedback loops that may be regarded as a fourth dimension of hierarchy in addition to strata, layers and echelons. The concept of a fourth dimension is a proposition that needs to be further researched; in particular, it needs to be contrasted against other possible dimensions of hierarchy. We have further illustrated the importance of feedback channels using a concrete example of a transformationsupporting management approach, namely EAM. The main limitation of this paper is that it is mostly conceptual. The conceptualizations, especially the multi-level feedback loops and their EAM implications need to be further analysed and transformed into useful innovative artefacts in future work. This could for example be performed by case studies in active enterprise transformation projects, or by reviewing evidence from past transformation. Nevertheless, this work provides a conceptual grounding of enterprise transformation on two well-established theories that can be used to derive requirements for concrete artefact construction.

\section{Acknowledgement}

We thank Antonia Albani for reviewing the manuscript and her valuable comments. This work has been supported by the Swiss National Science Foundation (SNSF). 


\section{References}

1. Rouse, W.B.: A Theory of Enterprise Transformation. Systems Engineering 8:4, 279-295 (2005)

2. Dietz, J.L.G., Hoogervorst, J.A.P.: Enterprise ontology in enterprise engineering. In: Proceedings of the 2008 ACM Symposium on Applied Computing, Fortaleza, Ceara, Brazil (2008)

3. Harmsen, F., Proper, E., Kok, N.: Informed Governance of Enterprise Transformations. In: Proper, E., Harmsen, F., Dietz, J.L.G. (eds.): Advances in Enterprise Engineering II, pp. 155-180. Springer, Berlin Heidelberg (2009)

4. Ross, J.W., Weill, P., Robertson, D.C.: Enterprise Architecture as Strategy. Creating a Foundation for Business Execution. Harvard Business School Press, Boston, MA (2006)

5. Tamm, T., Seddon, P.B., Shanks, G., Reynolds, P.: How Does Enterprise Architecture Add Value to Organisations? Communications Of The Association For Information Systems 28, 141-168 (2011)

6. Mesarovic, M.D.: Multilevel systems and concepts in process control. Proceedings of the IEEE 58:1, 111-125 (1970)

7. ISO/IEC/IEEE: Systems and software engineering -- Architecture description (ISO/IEC/IEEE 42010:2011) (2011)

8. Radeke, F.: Toward Understanding Enterprise Architecture Management's Role in Strategic Change: Antecedents, Processes, Outcomes. In: Proceedings of the 10th International Conference on Wirtschaftsinformatik WI 2.011, pp. 497-507 (2011)

9. Aier, S., Gleichauf, B., Winter, R.: Understanding Enterprise Architecture Management Design - An Empirical Analysis. In: Proceedings of the 10th International Conference on Wirtschaftsinformatik WI 2.011, pp. 645-654, Zurich (2011)

10. Strano, C., Rehmani, Q.: The Role of the Enterprise Architect. International Journal of Information Systems and e-Business Management 5:4, 379-396 (2007)

11. Winter, R.: Organisational Design and Engineering - Proposal of a Conceptual Framework and Comparison of Business Engineering with other Approaches. International Journal of Organizational Design and Engineering 1:1\&2, 126-147 (2010)

12. The Open Group.: TOGAF Version 9.1 (2011)

13. Dietz, J.L.G.: Architecture. Building strategy into design. Academic Service, The Hague (2008)

14. Österle, H., Brenner, W., Hilbers, K.: Total Information Systems Management - A European Approach. John Wiley \& Sons, Chichester (1993)

15. Åström, K.J., Murray, R.M.: Feedback Systems: An Introduction for Scientists and Engineers. Princeton University Press (2008)

16. Wholey, D.R., Brittain, J.: Characterizing Environmental Variation. The Academy of Management Journal 32:4, 867-882 (1989)

17. Child, J.: Organizational Structure, Environment and Performance: The Role of Strategic Choice. Sociology 6:1, 1-22 (1972)

18. Aveiro, D., Silva, A.R., Tribolet, J.: Towards a G.O.D. Organization for Organizational Self-Awareness. pp. 16-30. Springer, Berlin Heidelberg (2010)

19. Páscoa, C., Aveiro, D., Tribolet, J.: Organizational Configuration Actor Role Modeling Using DEMO. In: Practice-Driven Research on Enterprise Transformation - 4th Working Conference, PRET 2012, pp. 18-47. Springer, Gdask, Poland (2012)

20. Eisenhardt, K.M., Martin, J.A.: Dynamic Capabilities: What are They? Strategic Management Journal 21:10/11, 1105-1121 (2000) 
21. Pavlou, P.A., El Sawy, O.A.: The "Third Hand": IT-Enabled Competitive Advantage in Turbulence Through Improvisational Capabilities. Information Systems Research 21:3, 443$471(2010)$

22. Argyris, C., Schön, D.A.: Organizational learning: A theory of action perspective. AddisonWesley, Reading, Mass. (1978)

23. Páscoa, C., Tribolet, J.: Organizational and Design Engineering of the Operational and Support Components of an Organization: The Portuguese Air Force Case Study. pp. 47-77. Springer Berlin Heidelberg (2010)

24. Castela, N., Zacarias, M., Tribolet, J.: PROASIS: As-Is Business Process Model Maintenance. In: Harmsen, F., Grahlmann, K., Proper, E. (eds.): Practice-Driven Research on Enterprise Transformation, pp. 53-82. Springer Berlin Heidelberg (2011)

25. Buckl, S., Matthes, F., Roth, S., Schulz, C., Schweda, C.M.: A Conceptual Framework for Enterprise Architecture Design. In: 5th International Workshop on Trends in Enterprise Architecture Research (TEAR 2010), pp. 44-56. Springer, Delft (2010)

26. Deming, W.E.: Out of the Crisis. MIT Press, Cambridge (1986)

27. Teece, D.J., Pisano, G., Shuen, A.: Dynamic Capabilities and Strategic Management. Strategic Management Journal 18:7, 509-533 (1997)

28. Zollo, M., Winter, S.G.: Deliberate Learning and the Evolution of Dynamic Capabilities. Organization Science 13:3, 339-351 (2002)

29. Beer, S.: The Viable System Model: Its Provenance, Development, Methodology and Pathology. The Journal of the Operational Research Society 35:1, 7-25 (1984) 